\title{
PEATE AUTOMÁTICO EM RECÉM NASCIDOS DE RISCO: ESTUDO DA SENSIBILIDADE E ESPECIFICIDADE
}

\section{Automatic abr in newborns risk: study of sensitivity and specificity}

\author{
Rosanna Mariangela Giaffredo Angrisani ${ }^{(1)}$, Márcia Rumi Suzuki ${ }^{(2)}$, Gustavo Ribeiro Pifaia ${ }^{(3)}$, \\ José Ricardo Testa ${ }^{(4)}$, Elaine Colombo Sousa ${ }^{(5)}$, Daniela Gil ${ }^{(6)}$, Marisa Frasson de Azevedo ${ }^{(7)}$
}

\section{RESUMO}

Objetivo: verificar a sensibilidade e especificidade do teste de potencial evocado auditivo de tronco encefálico em equipamento automático (PEATEa), comparando-o ao teste de potencial evocado auditivo de tronco encefálico em equipamento diagnóstico (PEATE) em um programa de triagem auditiva neonatal em neonatos de risco. Método: foram avaliados 186 neonatos, 83 nascidos a termo e 103 pré-termo, sendo 88 do sexo masculino e 98 feminino. A triagem constou de emissões otoacústicas evocadas por estímulos transientes (EOAT), PEATEa e ao PEATE na mesma semana. Resultados: dos 186 neonatos avaliados, 156 (83,9\%) apresentaram audição normal. A perda condutiva foi encontrada em 9 neonatos (4,8\%), sendo 7 bilaterais e 2 unilaterais. A perda auditiva coclear foi observada em 5 neonatos $(2,7 \%)$ sendo um unilateral. Alteração central foi obtida em 11 neonatos $(5,9 \%)$ e um neonato foi diagnosticado como espectro da neuropatia auditiva -ENA $(0,5 \%)$.Em 4 casos houve atraso maturacional na avaliação inicial com normalização das respostas no mês seguinte Comparando-se os resultados do PEATEa com o PEATE, observou-se alta sensibilidade ( superior a 99\%) para identificação de perda coclear,condutiva, central, atraso maturacional e ENA .A especificidade do PEATEa foi de $100 \%$ para ENA, mediana para perda coclear ( $75 \%$ na OD e $60 \%$ na OE), e para alterações centrais (54,5\%OD e 63,6\% OE). Para identificação de perdas condutivas (inferior a 43\%). Conclusão: o PEATEa foi eficaz na identificação das neuropatias auditivas com elevada especificidade e sensibilidade. Contudo, falsos negativos foram observados para perdas cocleares, condutivas, para alterações centrais e atraso maturacional.

DESCRITORES: Recém-nascido; Triagem Neonatal; Potenciais Evocados Auditivos; Perda Auditiva

(1) Fonoaudióloga clínica do Hospital São Paulo - HSP; Especialista em Audiologia Clínica pelo Conselho Federal de Fonoaudiologia, São Paulo,SP, Brasil.

(2) Fonoaudióloga clínica; Doutoranda do Programa de Pós Graduação em Fonoaudiologia da Universidade Federal de São Paulo - UNIFESP, São Paulo,SP, Brasil.

(3) Médico Otorrinolaringologista. Especialista em Otologia pela Universidade Federal de São Paulo - UNIFESP São Paulo,SP, Brasil.

(4) Médico Otorrinolaringologista. Doutor, Professor Adjunto de Otorrinolaringologia da Universidade Federal de São Paulo - UNIFESP, São Paulo,SP, Brasil.

(5) Fonoaudióloga clínica do Hospital São Paulo -HSP; Mestranda em Disturbios da Comunicação Humana pela Universidade Federal de São Paulo - UNIFESP, São Paulo, SP, Brasil.

(6) Fonoaudióloga; Professora Adjunto do Departamento de Fonoaudiologia da Universidade Federal de São Paulo UNIFESP; Doutora em Ciências pela Universidade Federal de São Paulo - UNIFESP.

(7) Fonoaudióloga; Professor Associado da Escola Paulista de Medicina da Universidade Federal de São Paulo -

\section{INTRODUÇÃO}

A integridade anatômica e funcional do sistema auditivo é fator crítico para a aquisição de linguagem, uma vez que linguagem e audição se interrelacionam ${ }^{1}$. A audição se desenvolve no primeiro ano de vida, especialmente nos primeiros seis meses. Assim, é imperiosa a detecção precoce de qualquer tipo e grau de perda auditiva, bem como seu topodiagnóstico (alteração na porção periférica da via auditiva e/ou alterações ao longo da via auditiva central), possibilitando a intervenção imediata, minimizando o impacto negativo na linguagem ${ }^{2-6}$.

UNIFESP; Doutorado em Disturbios da Comunicação Humana pela Universidade Federal de São Paulo UNIFESP, São Paulo, SP, Brasil.

Conflito de interesses: inexistente 
A incidência de perda auditiva na população infantil varia de 1 a 3:1000 nascidos vivos sem risco e de 1 a 4:1000 recém-nascidos (RNs) atendidos em UTI Neonatal, segundo estudos epidemiológicos ${ }^{2,7,8}$.

A Triagem Auditiva Neonatal (TAN) compreende a realização de testes eletroacústicos e/ou eletrofisiológicos para a identificação da deficiência auditiva. Seu objetivo é o de distinguir na população em geral, os indivíduos que apresentam um distúrbio auditivo daqueles que não o apresentam. O critério em TAN é o de passa (sem probabilidade de perda) ou falha (com probabilidade de perda auditiva e necessidade de avaliação diagnóstica) ${ }^{2}$.

A escolha do teste é influenciada pela sua validade, que é conhecida pela sensibilidade e a especificidade do mesmo. Entende-se por Sensibilidade, a capacidade do teste de triagem identificar a perda auditiva, ou seja, das crianças deficientes auditivas da população testada, quantas foram identificadas pela TAN. A Especificidade é a capacidade de identificar o indivíduo ouvinte como normal, isto é, se a população que passou no teste era, de fato, audiologicamente normal ${ }^{9}$.

O teste de emissões otoacústicas evocadas (EOA) apresenta alta sensibilidade e especificidade (superior a $90 \%$ ), é mais rápido do que o potencial evocado auditivo de tronco encefálico (PEATE), levando aproximadamente um minuto por orelha, simples, de fácil aplicação e interpretação e tem sido bastante recomendado para TAN. A desvantagem é que não identifica as alterações retrococleares, que são comuns nos neonatos de UTI e sofrem maior interferência das alterações de orelha externa (como vérnix) e de orelha média (como as otites). A associação dos dois procedimentos (EOA e PEATE) de triagem em UTI neonatal já vem sendo recomendada pelo NIH (sigla em inglês para National Institute of Health) nos bebês que falharem nas EOA, antes da alta hospitalar ${ }^{10}$. Em 2007, JCIH (sigla em inglês para Joint Committe of Infant Hearing) recomendou a inclusão do PEATE nos neonatos que permanecerem UTI neonatal por mais de 5 dias, associado ao teste com as EOA ${ }^{7}$. A análise do custo-benefício também é recomendada por Porter et all ${ }^{11}$.

O protocolo de duas etapas com EOAT+ PEATE em equipamento automático (PEATEa) tem a vantagem de identificar as alterações retrococleares, sendo mais indicado para populações de alto risco. Como desvantagens, tem o custo mais elevado e maior tempo para TAN: aproximadamente 10 minutos $^{12}$. Este protocolo tem sido sugerido também como forma de reduzir os falso positivos. Em caso de falha nas EOAT e passa no PEATEa, a criança é liberada. Porém nesses casos corre-se o risco de, eventualmente, não identificar crianças com perda coclear em freqüências baixas, o que seria considerado um resultado falso negativo, já que o PEATE enfatiza as freqüências altas. Além disso, observa-se em alguns casos de perdas progressivas, crianças que falharam nas EOAT e passaram no PEATEa. Nesses casos, a ausência de EOAT, já sugeriria uma disfunção coclear inicial ${ }^{13}$.

O PEATE automático (PEATEa) é um procedimento de análise de tronco encefálico que tem como finalidade a TAN, a qual apresenta como resposta "Passa" ou "Falha", não permitindo a visualização das ondas pelo examinador ${ }^{14}$. É um procedimento mais barato, pois a análise da resposta é feita automaticamente pelo equipamento com critério estatístico de aprovação. No entanto, a literatura registra controvérsias quanto a sua sensibilidade e especificidade: alguns autores relatam sensibilidade de $100 \%$ e especificidade de $99,7 \%$ no PEATEa ${ }^{15}$, ao passo que outros registram sensibilidade de $40,9 \%$ e especificidade de $63,2 \%{ }^{16}$. Suppiej et al. referiram que o PEATE clínico é um teste de maior sensibilidade (100\%) e especificidade $(90,8 \%)$, quando comparado ao PEATEa que apresenta menor sensibilidade $(88,9 \%)$ e especificidade $(70,6 \%)^{17}$.

Considerando-se a recomendação de inclusão de PEATEa na triagem auditiva de neonatos de risco, o objetivo do presente estudo foi verificar sua sensibilidade e especificidade na TAN, comparando-o ao PEATE clínico em neonatos de risco atendidos no programa de TANU (triagem auditiva neonatal universal) do Hospital São Paulo.

\section{MÉTODO}

Trata-se de um estudo observacional e transversal realizado em neonatos de alto risco. A casuística do presente estudo foi composta por 186 neonatos (RN), sendo 83 nascidos a termo e 103 nascidos pré-termo, na maternidade do Hospital São Paulo vinculada à UNIFESP, no período de maio de 2009 a abril de 2010. Os neonatos foram selecionados após a leitura do prontuário do $\mathrm{RN}$ e anamnese com a mãe, identificando-se assim, os $\mathrm{RN}$ de alto risco.

Os responsáveis foram informados sobre os procedimentos do estudo e assinaram o termo de consentimento livre e esclarecido a fim de assegurar os preceitos éticos na realização de pesquisa com seres humanos.

Para a realização da TAN, a criança permaneceu no berço ou no colo da mãe, em sono natural.

Todos os RN foram submetidos à TAN com a captação das emissões otoacústicas evocadas por estímulo transiente (EOAT) e do PEATEa. A 
avaliação clínica completa foi realizada na mesma semana utilizando o PEATE no equipamento clínico, medidas de imitância acústica e exame otorrinolaringológico.

O preparo de todos os neonatos para a realização dos testes deu-se da seguinte forma: inspeção do meato acústico externo por meio de otoscópio marca Welch Allyn para visualização da membrana timpânica; limpeza da pele com pasta abrasiva (Nuprep) na região frontal (Fpz), occipital $(\mathrm{Oz})$ e na região zigomática para a colocação dos eletrodos para registro do PEATE no equipamento automático.

A captação das EOAT e PEATEa, foi realizada no equipamento automático portátil AccuscreenPRO marca GN Otometrics. Para iniciar os testes, a sonda auricular do AccuscreenPro foi posicionada no meato acústico externo do neonato para a emissão do estímulo acústico, sendo primeiramente feita a captação das EOAT e na sequencia, (após a fixação dos eletrodos) o PEATEa.

Para a realização das EOAT, o AccuscreenPro foi calibrado pelo fabricante para a análise automática por estatística binomial das respostas utilizando estímulo tipo clique não linear numa seqüência com velocidade de $60 \mathrm{~Hz}$ e intensidade de $70-84 \mathrm{~dB}$ NPS (45-60 dB NA), com auto-calibração dependendo do volume no meato acústico; espectro de freqüências de $1,4 \mathrm{KHz}$ a $4 \mathrm{KHz}$; velocidade de amostragem de $16 \mathrm{KHz}$ e artefato menor que $20 \%$ e estabilidade da sonda superior a $80 \%$. O registro de "PASSA" nas EOAT só é possível quando estes parâmetros são contemplados. Quando não são obtidos, o equipamento registra "FALHA".

No registro do PEATEa, após a verificação da impedância dos eletrodos (que deve ser menor que 6000 Ohms), o equipamento realiza a análise automática das respostas por meio da aplicação de estatística binomial. $O$ estímulo utilizado é o clique de $55 \mathrm{HZ}$ de velocidade e intensidade de $35 \mathrm{dBnNA}$, velocidade de amostragem de $10,2 \mathrm{KHz}$ e comprimento da imagem de 170 amostras/17ms. O EEG indica o potencial total registrado. Quando os parâmetros ideais são atingidos, o equipamento registra "PASSA".

O PEATE clínico foi realizado com o equipamento modelo Smart- EP, marca Intelligent Hearing Systems.

Para a realização do PEATE clínico, a limpeza da pele foi realizada em $\mathrm{Fpz}$ e nas mastóides direita e esquerda $\left(M_{2}\right.$ e $\left.M_{1}\right)$, para fixação dos eletrodos descartáveis (Meditrace 200 - marca Kendal), obedecendo à norma IES 10-20 (International Electrode System). Após a fixação dos eletrodos, o fone de inserção foi posicionado no meato acústico externo do neonato para a emissão do estímulo acústico. A captação do PEATE no equipamento clínico foi realizada nas intensidades de $80 \mathrm{dBNA}$ (pesquisa da integridade da via auditiva), $50 \mathrm{dBnNA}$ e 30dBnNA (limiar eletrofisiológico) para cliques.No caso de dúvida sobre a presença da onda $\mathrm{V}$ nas intensidades de $50 \mathrm{~dB}$ ou $30 \mathrm{dBnNA}$, aumentou-se a intensidade de 5 em $5 \mathrm{dBnNA}$, até a onda $V$ ser visualizada e reproduzida, garantindo a presença de potencial auditivo. $O$ estímulo acústico utilizado foi o clique de polaridade rarefeita, numa velocidade de apresentação de 27,7/seg, duração de 0,1 milissegundos, sendo empregado um total de 2000 estímulos com replicação dos traçados. A janela de gravação utilizada foi de $12 \mathrm{~ms}$.

Para a análise das respostas do PEATE clínico, foram medidas as latências absolutas das ondas I, III, V, e interpicos I-III, III-V, I-V a 80dBnNA para cliques e a latência da onda $V$ no limiar mínimo de intensidade pesquisado. O critério de normalidade considerado foi o indicado no equipamento SmartEP Intelligent Hearing.

As medidas de imitância acústica foram obtidas por meio do equipamento AT235 da Interacoustics. As curvas timpanométricas foram classificadas segundo critérios estabelecidos por Jerger ,1970 ${ }^{18}$. A otoscopia foi realizada pela equipe de otorrinolaringologistas da UNIFESP.

Os resultados foram analisados e a conclusão diagnóstica permitiu identificar:

- RNs com audição normal: EOAT presentes, timpanometria tipo A e PEATE com protocolo neurológico normal e limiar eletrofisiológico em 30dBNA.

- RNs com perda coclear: ausência de EOAT, com curva timpanométrica tipo A e PEATE com protocolo neurológico normal e limiar eletrofisiológico superior a $30 \mathrm{dBNA}$.

- RNs com perda condutiva: ausência de EOAT, com curva timpanométrica tipo $\mathrm{B}$ ou $\mathrm{C}$ e exame otorrinolaringológico alterado. PEATE com limiar eletrofisiológico aumentado e latências absolutas aumentadas com intervalos interpicos normais.

- RNs com alteração central: presença de EOAT, com aumento dos intervalos interpicos I-III, III-V e I-V no PEATE.

- RNs com atraso Maturacional quando se obteve um traçado com aumento de latências absolutas das ondas III e/ou V no primeiro PEATE, com normalização das mesmas no intervalo de 30 dias.

- RNs com Espectro da Neuropatia Auditiva: presença de EOAT, com ausência de respostas no PEATE e presença de microfonismo coclear. 
O projeto do estudo foi aprovado pelo Comitê de Ética e Pesquisa da Universidade Federal de São Paulo sob o no CEP 1362/08.

A análise estatística foi realizada empregandose o teste de Qui-Quadrado para medir o grau de relação e/ou associação entre o PEATE automático e clínico. Foram calculadas: Acurácia, Sensibilidade, Especificidade, Valor Preditivo Positivo e Negativo.
A sensibilidade e a especificidade medem a validade de um teste de diagnóstico. De acordo com a terminologia da saúde pública (Thorner e Remein, 1967), a precisão de um teste em identificar corretamente os pacientes positivos é chamada sensibilidade; sua precisão em classificar corretamente os pacientes negativos é chamada especificidade. O método para formular sensibilidade e especificidade está descrito na Figura abaixo.

\begin{tabular}{|c|c|c|c|}
\hline & Doente & Não Doente & Total \\
\hline Positivo & $\mathrm{A}$ & $\mathrm{B}$ & $\mathrm{A}+\mathrm{B}$ \\
\hline Negativo & $\mathrm{C}$ & $\mathrm{D}$ & $\mathrm{C}+\mathrm{D}$ \\
\hline Total & $\mathrm{A}+\mathrm{C}$ & $\mathrm{B}+\mathrm{D}$ & $\mathrm{A}+\mathrm{B}+\mathrm{C}+\mathrm{D}$ \\
\hline
\end{tabular}

Sensibilidade $=$ porcentagem de doentes designados como positivos com o teste de triagem $A /(A+C)$.

Especificidade $=$ porcentagem de não-doentes designados como negativos com o teste de triagem $D /(B+D)$.

Falso-positivo = B; Falso-negativo = C

Valor Preditivo Positivo $=A /(A+B)$

Valor Preditivo Negativo $=D /(C+D)$

Acurácia $=(A+D) /(A+B+C+D)$

\section{Figura 1 - Cálculo da Sensibilidade e Especificidade}

O nível de significância definido para o trabalho foi de $0,05(5 \%)$. Os intervalos de confiança ao longo do trabalho foram construídos com $95 \%$ de confiança estatística.

\section{RESULTADOS}

Dos 186 neonatos de risco avaliados, 156(83,9\%) apresentaram audição normal. A perda condutiva foi encontrada em 9 neonatos $(4,8 \%)$,sendo 7 bilaterais e 2 unilaterais. A perda auditiva coclear foi observada em 5 neonatos $(2,7 \%)$ sendo um unilateral. Alterações centrais foram verificadas em 11 neonatos $(5,9 \%)$ e um neonato foi diagnosticado como espectro da neuropatia auditiva (ENA) $(0,5 \%)$. Em quatro casos houve atraso maturacional na avaliação inicial (confirmados pela segunda avaliação, após 30 dias, com o PEATE - houve diminuição das latências absolutas das ondas I,III e $\mathrm{V}$ até os valores de normalidade constantes no manual do equipamento, com limiar eletrofisiológico normal).

O estudo da associação entre o resultado da triagem com PEATE automático e diagnóstico realizados com o PEATE clínico obtido por orelha nos neonatos com audição normal e perda coclear aparece descrito na Tabela 1. Os valores descritivos encontram-se na Tabela 2.

Houve relação estatisticamente significante em ambas as orelhas, ou seja, neonatos com audição normal passaram no PEATEa e neonatos com perda coclear não passaram. Observou-se valores altos para acurácia e sensibilidade, e medianos para especificidade.

O estudo da associação entre resultado da triagem com PEATE automático e diagnóstico realizado com o PEATE clínico obtido por orelha nos neonatos com audição normal e espectro da neuropatia auditiva aparece descrito na Tabela 3. Os valores descritivos encontram-se na Tabela 4.

Houve relação estatisticamente significante entre os resultados, com alta sensibilidade e especificidade. O único caso de espectro da neuropatia auditiva encontrado não passou na triagem com PEATEa. Apesar da estatística estar elevada, a análise deve ser cuidadosa, tendo-se em vista o número reduzido da amostra.

O estudo da associação entre resultado da triagem com PEATE automático e diagnóstico realizado com o PEATE clínico obtido por orelha nos neonatos com audição normal e alteração central aparece descrito na Tabela 5. Os valores descritivos encontram-se na Tabela 6.

Houve relação estatisticamente significante entre os resultados, com acurácia e sensibilidade elevadas, assim como os valores preditivos positivo e negativo. Entretanto, os valores de especificidade obtidos foram medianos.

O estudo da associação entre resultado da triagem com PEATE automático e diagnóstico realizado com o PEATE clínico obtido por orelha nos 
neonatos com audição normal e comprometimento condutivo aparece descrito na Tabela 7 . Os valores descritivos encontram-se na Tabela 8.

Houve relação estatisticamente significante entre os resultados em ambas as orelhas, porém com alta sensibilidade e baixa especificidade.
Os valores descritivos para neonatos com audição normal e atraso maturacional encontramse na Tabela 9.

Houve relação estatisticamente significante somente na orelha esquerda, com alta sensibilidade e muito baixa especificidade.

Tabela 1 - Relação e/ou Associação de PEATEa e PEATE por Orelha para neonatos com audição normal e perda coclear

\begin{tabular}{cccccccc}
\hline & & \multicolumn{2}{c}{ Normal } & \multicolumn{2}{c}{ Coclear } & \multicolumn{2}{c}{ Total } \\
\cline { 3 - 8 } & & $\mathbf{N}$ & $\%$ & $\mathbf{N}$ & $\%$ & $\mathbf{N}$ & $\%$ \\
\hline \multirow{2}{*}{ OD } & Passou & 155 & $99 \%$ & 1 & $25 \%$ & 156 & $98 \%$ \\
& Falhou & 1 & $1 \%$ & 3 & $75 \%$ & 4 & $3 \%$ \\
\hline \multirow{2}{*}{ OE } & Passou & 157 & $100 \%$ & 2 & $40 \%$ & 159 & $98 \%$ \\
& Falhou & 0 & $0 \%$ & 3 & $60 \%$ & 3 & $2 \%$ \\
\hline
\end{tabular}

Tabela 2 - Medidas descritivas para neonatos com audição normal e perda coclear

\begin{tabular}{lcc}
\hline & OD & OE \\
\hline P-valor & $<0,001$ & $<0,001$ \\
Acurácia & $98,8 \%$ & $98,8 \%$ \\
Sensibilidade & $99,4 \%$ & $100,0 \%$ \\
Especificidade & $75,0 \%$ & $60,0 \%$ \\
VP + & $99,4 \%$ & $98,7 \%$ \\
VP - & $75,0 \%$ & $100,0 \%$ \\
\hline
\end{tabular}

Tabela 3 - Relação e/ou Associação de PEATEa e PEATE por orelha para neonatos com audição normal e espectro da neuropatia auditiva

\begin{tabular}{cccccccc}
\hline & & \multicolumn{2}{c}{ Normal } & \multicolumn{2}{c}{ Neuropatia } & \multicolumn{2}{c}{ Total } \\
\cline { 3 - 8 } & & $\mathbf{N}$ & $\%$ & $\mathbf{N}$ & $\%$ & $\mathbf{N}$ & $\%$ \\
\hline \multirow{2}{*}{ OD } & Passou & 155 & $99 \%$ & 0 & $0 \%$ & 155 & $99 \%$ \\
& Falhou & 1 & $1 \%$ & 1 & $100 \%$ & 1 & $1 \%$ \\
\hline \multirow{2}{*}{ OE } & Passou & 156 & $100 \%$ & 0 & $0 \%$ & 156 & $99 \%$ \\
& Falhou & 0 & $0 \%$ & 1 & $100 \%$ & 1 & $1 \%$ \\
\hline
\end{tabular}

Tabela 4 - Medidas descritivas para neonatos com audição normal e espectro da neuropatia auditiva

\begin{tabular}{lcc}
\hline & OD & OE \\
\hline P-valor & $<0,001$ & $<0,001$ \\
Acurácia & $99,4 \%$ & $100,0 \%$ \\
Sensibilidade & $99,4 \%$ & $100,0 \%$ \\
Especificidade & $100,0 \%$ & $100,0 \%$ \\
VP + & $100,0 \%$ & $100,0 \%$ \\
VP - & $50,0 \%$ & $100,0 \%$ \\
\hline
\end{tabular}


Tabela 5 - Relação e/ou Associação de PEATEa e PEATE por orelha para neonatos com audição normal e alteração central

\begin{tabular}{cccccccc}
\hline & & \multicolumn{2}{c}{ Normal } & \multicolumn{2}{c}{ Central } & \multicolumn{2}{c}{ Total } \\
\cline { 3 - 7 } & & $\mathbf{N}$ & $\%$ & $\mathbf{N}$ & $\%$ & $\mathbf{N}$ & $\%$ \\
\hline \multirow{2}{*}{ OD } & Passou & 155 & $99 \%$ & 5 & $45 \%$ & 160 & $96 \%$ \\
& Falhou & 1 & $1 \%$ & 6 & $55 \%$ & 7 & $4 \%$ \\
\hline \multirow{2}{*}{ OE } & Passou & 156 & $100 \%$ & 4 & $36 \%$ & 160 & $96 \%$ \\
& Falhou & 0 & $0 \%$ & 7 & $64 \%$ & 7 & $4 \%$ \\
\hline
\end{tabular}

Tabela 6 - Medidas descritivas para neonatos com audição normal e alteração central

\begin{tabular}{lcc}
\hline & OD & OE \\
\hline P-valor & $<0,001$ & $<0,001$ \\
Acurácia & $96,4 \%$ & $97,6 \%$ \\
Sensibilidade & $99,4 \%$ & $100,0 \%$ \\
Especificidade & $54,5 \%$ & $63,6 \%$ \\
VP + & $96,9 \%$ & $97,5 \%$ \\
VP - & $85,7 \%$ & $100,0 \%$ \\
\hline
\end{tabular}

Tabela 7 - Relação e/ou Associação de PEATEa e PEATE por Orelha para neonatos com audição normal e comprometimento condutivo

\begin{tabular}{cccccccc}
\hline & & \multicolumn{2}{c}{ Normal } & \multicolumn{2}{c}{ Condutivo } & \multicolumn{2}{c}{ Total } \\
\cline { 3 - 8 } & & $\mathbf{N}$ & $\%$ & $\mathbf{N}$ & $\%$ & $\mathbf{N}$ & $\%$ \\
\hline \multirow{2}{*}{ OD } & Passou & 155 & $99 \%$ & 6 & $67 \%$ & 161 & $98 \%$ \\
& Falhou & 1 & $1 \%$ & 3 & $33 \%$ & 4 & $2 \%$ \\
\hline \multirow{2}{*}{ OE } & Passou & 156 & $100 \%$ & 4 & $57 \%$ & 160 & $98 \%$ \\
& Falhou & 0 & $0 \%$ & 3 & $43 \%$ & 3 & $2 \%$ \\
\hline
\end{tabular}

Tabela 8 - Medidas descritivas para neonatos com audição normal e comprometimento condutivo

\begin{tabular}{lcc}
\hline & OD & OE \\
\hline P-valor & $<0,001$ & $<0,001$ \\
Acurácia & $95,8 \%$ & $97,5 \%$ \\
Sensibilidade & $99,4 \%$ & $100,0 \%$ \\
Especificidade & $33,3 \%$ & $42,9 \%$ \\
VP + & $96,3 \%$ & $97,5 \%$ \\
VP - & $75,0 \%$ & $100,0 \%$ \\
\hline
\end{tabular}

Tabela 9 - Medidas descritivas para neonatos com audição normal e atraso maturacional

\begin{tabular}{lcc}
\hline & OD & OE \\
\hline P-valor & 0,872 & $<0,001$ \\
Acurácia & $96,9 \%$ & $97,5 \%$ \\
Sensibilidade & $99,4 \%$ & $100,0 \%$ \\
Especificidade & $0,0 \%$ & $20,0 \%$ \\
VP + & $97,5 \%$ & $97,5 \%$ \\
VP - & $0,0 \%$ & $100,0 \%$ \\
\hline
\end{tabular}




\section{DISCUSSÃO}

A ocorrência de perda coclear observada no presente estudo (Tabela 1) coincidiu com os estudos de Suppiej et al que observaram 2,9\% de perda auditiva congênita em $\mathrm{RN}$ de risco ${ }^{17}$. Tal ocorrência foi similar à descrita na literatura para recém nascidos de UTI neonatal (3\% a 5\%) ${ }^{3}$. De fato, desde 1999, a Academia Americana de Pediatria já relatara incidência de $2 \%$ a $4 \%$ de perda auditiva em neonatos de risco ${ }^{19}$. Outros autores também verificaram ocorrência de perda neurossensorial entre $2,2 \%$ e 3,2\% na população de UTI neonatal 20,21, estando em concordância com os achados do presente estudo.Lima et al encontraram $8,33 \%$ de crianças com perda auditiva bilateral ${ }^{22}$.

No presente estudo, dos 186 neonatos avaliados, $122(65,6 \%)$ permaneceram em UTI neonatal por mais de 5 dias e destes, 5 (4\%) apresentaram perda auditiva coclear. Estes resultados concordam com os de Uus et al que estudaram 169.487 bebês e destes, 169 tiveram diagnóstico de surdez permanente, sendo que $50 \%$ destes bebes eram provenientes de UTI neonatal ${ }^{23}$.

Pereira et al, observaram maior ocorrência de perda auditiva em neonatos pré-termo $(3,1 \%)$ em relação aos nascidos a termo $(0,8 \%)^{24}$. No presente estudo, dos 186 neonatos avaliados, 103 (55,3\%) nasceram pré-termo e destes, dois $(1,95 \%)$ apresentaram perda coclear. Desta forma, a ocorrência de perda auditiva coclear observada foi similar à relatada na literatura.

O estudo da associação entre a triagem auditiva com PEATEa e os resultados do PEATE clínico, demonstrou relação estatisticamente significante em ambas orelhas (tabela 2), com valores elevados de acurácia $(98,8 \%)$ e de sensibilidade $(99,4 \%$ para orelha direita e $100 \%$ para orelha esquerda). A alta sensibilidade do PEATE clínico na identificação de perda auditiva coclear (tabela 2) também foi observada por Hall et al que, avaliando 300 neonatos, obtiveram $100 \%$ de sensibilidade ${ }^{15}$. Hermann et al obtiveram $98 \%$ de sensibilidade, utilizando o PEATEa $^{25}$. Suppiej et al encontraram sensibilidade do PEATEa, com o mesmo equipamento do presente estudo, de $88,9 \%{ }^{17}$.

Entretanto, observou-se valores de especificidade medianos (de $60 \%$ e $75 \%$ ) visto que três orelhas com perda coclear passaram na triagem com PEATEa e falharam nas EOAT apresentando limiares eletrofisiológicos elevados nas freqüências baixas ( perda auditiva ascendente) identificada com utilização do estímulo tone burst no PEATE clinico.

Estudos tem demonstrado uma maior correlação dos limiares eletrofisiológicos com as freqüências altas. Matas referiu que a pesquisa dos limiares eletrofisiológicos permite identificar o grau da perda somente na região do espectro do estímulo - com o clique, os limiares coincidem mais com a região de 2000 a 4000 ou de 3000 a $6000 \mathrm{~Hz}^{26}$, dependendo do equipamento utilizado. $O$ uso do PEATE com tone burst no diagnóstico de neonatos vem sendo recomendado na literatura para caracterizar a configuração do audiograma ${ }^{27}$.

Hall et al observaram maior especificidade $(99,7 \%)$ do que a obtida no presente estudo ${ }^{15}$. Tal diferença poderia ser atribuída a diferenças metodológicas e de equipamento com diferentes velocidades de estimulação e uso de critérios estatísticos de passa utilizando Fsp maior ou igual a 3,2 ou pelo fato da não ocorrência de perdas auditivas cocleares ascendentes, que são mais raras.

No presente estudo, o valor preditivo positivo foi superior a $98 \%$, ou seja, se a criança falhar na triagem com o PEATEa, terá mais de $98 \%$ de chance de apresentar perda auditiva coclear. O valor preditivo negativo foi menor para a orelha direita $(75 \%)$ em relação à orelha esquerda (100\%).

$O$ uso do PEATEa em neonatos de risco tem sido recomendado por gerar menor índice de encaminhamentos para diagnóstico ${ }^{28}$. Entretanto,a possibilidade de não identificar perdas cocleares ascendentes poderia se confrontar com a vantagem da reduzida taxa de encaminhamentos.

A triagem auditiva realizada com PEATEa foi bastante sensível para identificação do espectro da neuropatia auditiva (ENA). O único caso de ENA foi identificado pelo PEATEa (Tabela 3). Houve relação estatisticamente significante entre os resultados com elevada sensibilidade e especificidade. A acurácia foi superior a $99 \%$ com valores de sensibilidade superiores a $99 \%$ e especificidade de $100 \%$. Portanto, o uso do PEATEa em crianças de alto risco foi eficaz na identificação do espectro da neuropatia auditiva com valores elevados de sensibilidade e especificidade (Tabela 4).

Apesar da estatística estar elevada, a análise deve ser realizada com cuidado, tendo em vista o número reduzido da amostra. A baixa ocorrência de ENA $(0,5 \%)$ observada no presente estudo, já era esperada, estando de acordo com dados na literatura, que tem relatado ocorrência do ENA na população geral como sendo de $0,01 \%^{24}$ a $0,04 \%^{29}$. Ngo et al já haviam descrito uma prevalência de 0,06\% de neuropatia auditiva em triagem universal ${ }^{30}$. A prevalência de ENA em população de alto risco varia de $0,2 \%{ }^{30}$ a $4 \%{ }^{31}$. Berlin et al. encontraram $2 \%$ de ENA em bebês de UTI neonatal ${ }^{32}$.

Tal prevalência é maior entre as crianças com perda auditiva: $4 \%$ a $8 \%$ das crianças com perda auditiva de grau profundo apresentaram ENA, 
segundo estudo de Foerst et al. ${ }^{33}$. A prevalência de ENA também aumenta em neonatos de risco em especial nos casos de hiperbilirrubinemia ${ }^{29,30}$.

De fato a maior ocorrência do ENA em neonatos de risco, levou o $\mathrm{JCIH}$ a recomendar a inclusão do PEATE na triagem de RN que permaneceram por mais de cinco dias em UTI ${ }^{7}$.

O estudo da associação entre os resultados da TAN com PEATEa e PEATE clínico nos neonatos normais e com alterações centrais demonstrou relação significante, com acurácia superior a 96\% e sensibilidade superior a 99,4\% (tabelas 5 e 6). O uso do PEATEa identificou mais de $99,4 \%$ das alterações centrais com valor preditivo positivo de 97\% ,ou seja, se o neonato falhar no PEATEa terá 97\% de chance de apresentar alteração central. Entretanto, os valores de especificidade foram medianos $(54,5 \%-63,6 \%)$. Nove orelhas das 320 que passaram na TAN com PEATEa, apresentaram alteração central na avaliação diagnóstica, caracterizada por aumento das latências absolutas das ondas III e/ou V sem melhora na segunda avaliação em trinta dias, e interpicos I-III, III-V e/ou I-V aumentados. Apesar disso, o valor preditivo negativo variou de $85,7 \%$ a $100 \%$ ou seja, o neonato que passou na triagem com PEATEa teve de $85 \%$ a $100 \%$ de chance de ser audiologicamente normal.Suppiej et al. também registraram diferenças de sensibilidade e especificidade comparando o PEATEa e PEATE ${ }^{17}$.

Apesar do PEATEa e PEATE investigarem a mesma via neural , as diferenças encontradas poderiam ser atribuídas a diferenças metodológicas. Em ambos os procedimentos, o estímulo clique é apresentado a $35 \mathrm{dBnNA}$ porém em velocidades de apresentação diferentes, sendo a velocidade de apresentação do PEATEa de $55 \mathrm{~Hz}$ e do PEATE clínico, de 27,7/mseg. Em RN de risco para alteração neurológica ou prematuros tais parâmetros poderiam influenciar na captação das respostas.

Além disso, a análise automática da resposta baseia-se em comparação com onda obtida na latência usual que poderia diferir em prematuros, principalmente com velocidade elevada de apresentação do estímulo. Jacobson et al. ${ }^{34}$ encontraram maior compatibilidade entre PEATEa e clínico utilizando o equipamento Algo I cuja velocidade de apresentação é de $37 / \mathrm{mseg}$, inferior à utilizada pelo equipamento Accuscreen utilizado no presente estudo. Esta hipótese parece ter sido confirmada pelo estudo de Van Straaten et al. em 1996 que obtiveram elevada sensibilidade (100\%) e especificidade (94\%) com o equipamento Algo I ${ }^{35}$.

Em relação ao comprometimento condutivo, houve relação significante entre os resultados em ambas as orelhas, porém com alta sensibilidade e baixa especificidade (Tabelas 7 e 8 ). A especificidade encontrada foi de $33,3 \%$ e $42,9 \%$ para as orelhas direita e esquerda respectivamente.O fato de deixar passar na triagem as perdas condutivas gera menor índice de encaminhamentos e por esta razão o uso do PEATEa tem sido considerado como vantajoso ${ }^{28}$. Entretanto, Doyle et al. observaram em estudo prospectivo que o neonato que falha na triagem por alteração de orelha média tem mais chance de apresentar otites de repetição no primeiro ano de vida ${ }^{36}$. No Brasil, Pereira \& Azevedo observaram que o neonato que falha por alteração de orelha média tem 2,9 vezes mais chance de ter alterações condutivas no primeiro ano de vida ${ }^{37}$. Como as otites de repetição no primeiro ano são consideradas como fator de risco para alteração de processamento auditivo, a vantagem de não se identificar as alterações condutivas poderia ser questionada.

No que se refere ao atraso na maturação da via auditiva, o PEATEa mostrou-se muito sensível $(99,4 \%$ para orelha direita e $100 \%$ para a orelha esquerda), porém com especificidade extremamente baixa ( $0 \%$ para orelha direita e $20 \%$ para a orelha esquerda), ou seja, neonatos com alterações transitórias no PEATE clínico passaram na triagem por PEATEa(tabela 9). Como as quatro crianças apresentaram resultados compatíveis com a normalidade na reavaliação após 30 dias, a não identificação do atraso mostrou-se irrelevante do ponto de vista clínico.

Nos programas de TAN, recomenda-se um índice de respostas falso positivo inferior a $4 \%{ }^{7}$

No presente estudo, no que se refere à identificação da perda auditiva coclear, houve baixa ocorrência de falsos positivos (1/312- $0,3 \%)$, concordando com estudo de Freitas et al. ${ }^{28}$.

Os falsos positivos verificados no presente estudo foram inferiores a $0,6 \%$. Valores de falsopositivo, inferiores a $3 \%$, tem sido recomendados visto que, em altas taxas, além de gerar ansiedades familiares desnecessárias, aumentam o número de retornos, elevando o custo do programa de triagem $^{9,19}$.

Três orelhas com perda coclear passaram na triagem com PEATEa, caracterizando a presença de falsos negativos ( $3 / 312-0,9 \%$ ).

Suppiej et al. ${ }^{17}$ analisando três protocolos de triagem aplicados em 151 neonatos de risco, encontraram falsos negativos $(11,1 \%)$ apenas no protocolo que utilizou PEATEa isolado.

A possibilidade da ocorrência de falsos negativos na TAN com PEATEa já havia sido relatada por Johnson et al. ,que encontrou 21 crianças que falharam na triagem com EOAT e passaram no PEATEa com perda auditiva coclear identificadas 
no acompanhamento. Como a avaliação completa não foi realizada ao nascimento, a hipótese de perda adquirida não poderia ser descartada e por esta razão, o estudo de Johnson recebeu críticas ${ }^{38}$.

Entretanto, a ausência de EOA no primeiro teste realizado poderia significar a presença de uma disfunção coclear ao nascimento a qual poderia evoluir para perda auditiva coclear ${ }^{13}$. Além disso, perdas auditivas ascendentes poderiam não ter sido identificadas pelo PEATEa similarmente ao verificado no presente estudo.

Para os RNs com alteração central, valores elevados de falso-negativo foram encontrados, o mesmo ocorrendo para os RNs com comprometimento condutivo (Tabelas 6 e 8).

O desejável seria não haver falsos negativos ${ }^{9,19}$, pois isto contraria o objetivo principal da TAN, que é o de detectar possíveis alterações auditivas, possibilitando a intervenção e a habilitação precoces a fim de garantir um bom desenvolvimento global da criança.

\section{CONCLUSÃO}

O PEATEa foi eficaz para a identificação das neuropatias auditivas com elevada especificidade e sensibilidade. Contudo, falsos negativos foram observados para perdas cocleares, condutivas, alterações centrais e atraso maturacional.

\section{AGRADECIMENTOS}

À Fundação de Amparo à Pesquisa do Estado de São Paulo - FAPESP pelo financiamento deste estudo, sem o qual não haveria a possibilidade de realização.

Ao Hospital São Paulo por ceder espaço físico adequado à realização dos procedimentos.

\section{ABSTRACT}

Purpose: to study the sensitivity and specificity of automatic ABR in comparison to diagnostic ABR of newborns under risk in a neonatal hearing screening program. Method: one hundred and eighty six neonates were evaluated, 83 born at term and 103 were pre terms, 88 male and 98 female gender. Evaluation procedures consisted of transient otoacoustic emissions(TOAEs), A-ABR and diagnostic ABR carried out in the same week. Results: from the 186 evaluated newborns, 156 (83.9\%) showed normal hearing. Conductive hearing loss was verified in nine neonates $(4.8 \%)$, seven bilateral and two unilateral. Cochlear hearing loss was observed in five neonates $(2.7 \%)$, one unilateral and four bilateral. Central evidences were observed in eleven neonates $(5.9 \%)$ and one neonate was diagnosed with auditory neuropathy spectrum disorder $(0,5 \%)$. In four cases, we found a maturational delay in the first evaluation with normal result within one month. In the comparison of A-ABR with diagnostic ABR, high sensitivity rates were observed (99\% and higher) in the identification of cochlear, conductive, central and auditory neuropathy. Specificity rate of A-ABR was $100 \%$ for auditory neuropathy, $75 \%$ for right ear and $60 \%$ for left ear cochlear hearing losses and $54.5 \%$ for right ear and $63.6 \%$ for the left ear central abnormalities. For conductive hearing losses, specificity was low (lower than $43 \%$ ). Conclusion: A-ABR was efficient in the identification of auditory neuropathies with high sensitivity and specificity rates. However, false negative responses were observed in the group diagnosed with cochlear and conductive hearing losses, central abnormalities and maturational delay.

KEYWORDS: Newborn; Neonatal Screening; Evoked Potentials, Auditory; Hearing Loss

\section{REFERÊNCIAS}

1. Azevedo MF. Programa de Prevenção e Identificação Precoce dos Distúrbios da Audição. In: Schochat E.Processamento Auditivo.São Paulo, Lovise, 1996. 75-105.

2. Lewis DR, Marone SAM, Mendes BCA, Cruz OLM, Nóbrega M. Comitê multiprofissional em saúde auditiva . COMUSA Braz J Otorhinolaryngol. 2010;76 (1):121-8.

3. Hilú MRPB, Zeigelboim BS. O conhecimento,a valorização da triagem auditiva neonatal e a intervenção precoce da perda auditiva. Rev CEFAC.2007; 9(4):563-70.

4. Yoshinaga-Itano C, Sedey AL, Coulter DK, Mehl AL. Language of early-and later-identified children with hearing loss. Pediatrics. 1998;102(5):1161-71. 
5. Wrightson AS. Universal Newborn Hearing Screening. Am Fam Physician 2007;75:1349-52.

6. Chiong C, Ostrea E Jr, Reyes A, et al. Correlation of hearing screening with developmental outcomes in infants over a 2-year period. ActaOtolaryngol. 2007;127: 384-8.

7. Joint Committee on Infant Hearing, Year 2007 Position Statement: Principles and Guidelines for Early Hearing Detection and Intervention Programs. J Pediatr.. 2007; 120(4): 898-921.

8. Hanna,K F, Maia RA. Triagem auditiva neonatal: incidência de deficiência auditiva neonatal sob a perspectiva da nova legislação paulista.Rev.Bras. saude matern.infant.2010;10(2):257-64.

9. Bess, FH; Humes, LE. Fundamentos de Audiologia. 2ed. Porto Alegre: Artmed, 1998. cap. 2, p. 35-63.

10. NIH - National Institute of Health.1993 disponível em http://www.nlm.nih.gov/medlineplus

11. Porter HL, Neely ST, Gorga MP.Using benefitcost ratio to select Universal Newborn Screening test criteria. Ear Hear. 2009; 30(4): 447-57.

12. Monteiro PC. Triagem Auditiva Neonatal: 0 custo $X$ efetividade de cinco protocolos em uma maternidade de São Paulo. São Paulo: Pontifícia Universidade Católica; 2008 [dissertação]

13. Azevedo MF.Triagem Auditiva Neonatal. In: Tratado de Fonoaudiologia/ [org] Fernandes FDM;Mendes BCA; Navas ALGP. $2^{\text {a }}$ ed.-São Paulo: Roca, 2009.

14. Ito-Orejas JI, Ramirez B, Morais D, Almaraz A, Fernandez-Calvo JL. Comparison of two-step transient evoked otoacoustic emissions (TEOAE) and automated auditory brainstem response (AABR) for universal newborn hearing screening programs. Intern J of Ped Otorhinolar. 2008;72:1193-201.

15. Hall III J.W, Smith S.D, Popelka G.R - Newborn Hearing Screening with Combined Otoacoustic Emissions and Auditory Brainstem Responses. J Am.Acad. Audiol 2004; 15:414-25.

16. Boo NY;Rohani AJ;Asma A. Detection of sensorineural hearing loss using automated auditory brainstem-evoked response and transientevoked otoacoustic emission in term neonates with severe hyperbilirubinaemia. Singapure Med J. 2008; 49(3):209-13.

17. Suppiej A, Rizzardi E, Zanardo V, Franzoi M, Ermani M, Orzan E. Reliability of hearing screening in high-risk neonates:comparative study of otoacoustic emission, automated and conventional auditory brainstem response. Clinical Neurophysiology, 2007; 118(4), 869-76.

18. Jerger J. Clinical experience with impedance audiometry. Arch Otolaryngol,1970; 92(4):311-24.
19. American Academy of Pediatrics. Task force on newborn and infant hearing. Pediatrics. 1999; 103(2): 527-9.

20. White KR, Vohr BR, Meyer S, Widen, JE, Johnson JL, Gravel, JS et al. A multisite study to examine the efficacy of the otoacoustic emission/ automated auditory brainstem response newborn hearing screening protocol: Research design and results of the study. A.m J. of Audiol. 2005;14, S186-S99.

21. Lima GML. Análise da triagem auditiva por audiometria automática de tronco encefálico de recém-nascidos internados em unidade de cuidados intensivos e intermediários. Rev. Ciênc. Méd. 2005; 14(2): 147-56.

22. Lima MCMP, Rossi TRF, Françozo MFC, Marba ST, Lima GML, Santos MFC. Detecção de perdas auditivas em neonatos de um hospital público Rev Soc Bras Fonoaudiol. 2010; 15(1):1-6

23. Uus K, Bamford J. Effectiveness of PopulationBased Newborn Hearing Screening in England: Ages of Interventions and Profile of Cases. Pediatrics 2006; 117:887-93.

24. Pereira PKS, Martins AS, Vieira MR, Azevedo, MF de. Programa de triagem auditiva neonatal: associação entre perda auditiva e fatores de risco. Pró-Fono Revista de Atualização Científica. 2007;19( 3) ; 267-78.

25. Herrmann BS, Thornton AR, Joseph JM. Automated infant hearing screening using the ABR: development and validation. Am J Audiol. 1995; 4:6-14.

26. Matas CG; Neves IF. Potenciais Evocados Auditivos de Curta Latêncialn: Tratado de Fonoaudiologia/[org]Fernandes FDM;Mendes BCA; Navas ALGP. 2 ${ }^{a}$ Ed.-São Paulo: Roca, 2009.

27. Ribeiro FM; Carvallo RMM. Tone evoked ABR in normal hearing neonates. Int J Audiol.2008;47:21-9. 28. Freitas VS, Alvarenga KF, Bevilacqua MC, Martinez MAN, Costa OA. Critical analysis of three newborn hearing screening protocols (original title: Análise crítica

de três protocolos de triagem auditiva neonatal). Pró-Fono Revista de Atualização Científica. 2009; 21(3):201-6.

29. Kirkim,G; Serbetcioglu,B; Erdag TK et al. The frequency of neuropathy detected by universal newborn hearing screening program. Int J Pediatric Otorhinolaryngol 2008;72:1461-9.

30. Ngo,RYS Tan HKK Balakrishnan A et al Auditory neuropathy/auditory dys-sincrony detected by universal newborn screening. Int $\mathrm{J}$ Pediatric Otorhrinolayngol 2006; 70:1299-306.

31. Stein L K, Tremblay K, Pasternak J, Banerjee $\mathrm{S}$, Lindemann K, Kraus N. Brainstem abnormalities 
in neonates with normal otoacoustic emissions. Semin Hear 1996; 17:197-213.

32. Berlin C, Hood L, Rose K. On renaming auditory neuropathy as auditory dys-synchrony. Audiol Today. 2001; 13:15-7.

33. Foerst $A$; Beutner,D;Lang-Roth $R$ et al Prevalence of auditory neuropathy/synaptopathy in a population of children with profound hearing loss. Int J Pediatric Otorhrinolayngol. 2006;70: 1415-22.

34. Jacobson JT, Jacobson CA, Spahr RC. Automated and conventional ABR screening techniques in high risk infants. J Am Acad Audiol. 1990; 1 (4):187-95.

35. Van Straaten HL Groote ME, Oudesluys-Murphy AM. Evaluation of an automated auditory brainstem response infant hearing screening method in at risk neonates. Eur J Pediatr. 1996; 155:702-5.
36. Doyle KJ; Kong, YY; Strobel K.; Dallaire,P; Ray, RM. Neonatal middle ear effusion predicts chronic otitis media with effusion. Otol. Neurotol. 2004; 25(3): 318-22.

37. Pereira PKS; Azevedo, MF de; Testa JR. Conductive impairment in newborn who failed the newborn hearing screening. Braz J Otorhinolaryngol. 2010;76(3):347-54.

38. Johnson, J. L., White, K. R., Widen, J. E., Gravel, J. S., Vohr,B. R., James, M., et al. A multisite study to examine the efficacy of the otoacoustic emission /automated auditory brainstem response newborn hearing screening protocol: Introduction and overview of the study. Am J of Audiol. 2005; 14:S178-S85.
http://dx.doi.org/10.1590/S1516-18462011005000065

RECEBIDO EM: 22/09/2010

ACEITO EM: 01/02/2011

Endereço para correspondência:

Rosanna M.Giaffredo Angrisani

Rua Martiniano de Carvalho, 548/41

São Paulo - São Paulo

CEP: 01321-000

E-mail: roangrisani@gmail.com 